\title{
Potential Consequences of Sars-Cov-2 Pandemic on Birth Rates and Subsequent Demographics
}

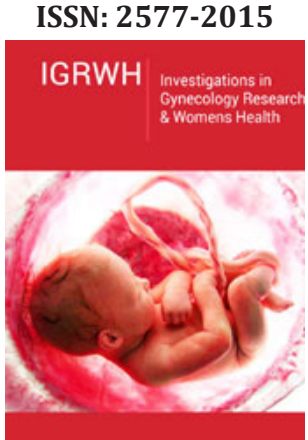

*Corresponding author: Léo Pomar, Department Woman-Mother-Child, Lausanne University Hospital, Switzerland, Email: leo.pomar@chuv.ch

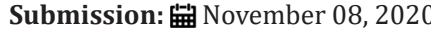

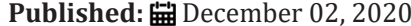

Volume 3 - Issue 5

How to cite this article: Léo Pomar, Agathe Contier, Jeffrey E Harris, Guillaume Favre, Karin NielsenSaines, David Baud. Potential Consequences of Sars-Cov-2 Pandemic on Birth Rates and Subsequent Demographics. Invest Gynecol Res Women's Health. 3(5). IGRWH. 000574. 2020.

DOI: 10.31031/IGRWH.2020.03.000574

Copyright@ Léo Pomar, This article is distributed under the terms of the Creative Commons Attribution 4.0 International License, which permits unrestricted use and redistribution provided that the original author and source are credited.
Léo Pomar $^{1 *}$, Agathe Contier ${ }^{1}$, Jeffrey E Harris ${ }^{2}$, Guillaume Favre ${ }^{1}$, Karin Nielsen-Saines ${ }^{3}$ and David Baud ${ }^{1}$

${ }^{1}$ Department Woman-Mother-Child, Lausanne University Hospital, Switzerland

${ }^{2}$ Department of Economics, Massachusetts Institute of Technology, USA

${ }^{3}$ Division of Pediatric Infectious Diseases, David Geffen School of Medicine at UCLA, Los Angeles, USA

\section{Opinion}

The SARS-CoV 2 pandemic is now responsible for over 48 million infections and one million deaths worldwide. Pandemics impact population growth not only by excessive mortality, but also by preventing births. In previous pandemics occurring in the $20^{\text {th }}$ and $21^{\text {st }}$ centuries, a trend towards birth rate reduction was observed 9 months later (Figure 1). After the 1918-20 HIN1 influenza pandemic, birth rates dropped by 5 to $15 \%$ in comparison to average rates before the pandemic [1]. This drop was related to higher death rates in pregnant patients (case fatality rate of $27 \%$ ), as well as miscarriages and stillbirths (increased by $0.4 \%$ and $2.2 \%$ respectively as compared to prior average rates) [1,2]. A rebound in birth rates, however, occurred 1-2 years after this decline [3]. Moreover, several studies have shown that children born in 1919, and thus exposed to the H1N1 virus in utero, experienced worse health and socioeconomic outcomes in older ages than surrounding birth cohorts [4]. SARS-CoV and Ebola were also associated with similar trends in birth rates during and after the pandemics in 2004 and 2015, respectively. In Brazil, the Zika virus pandemic led to a 10 to 20\% drop-in birth rates [5].

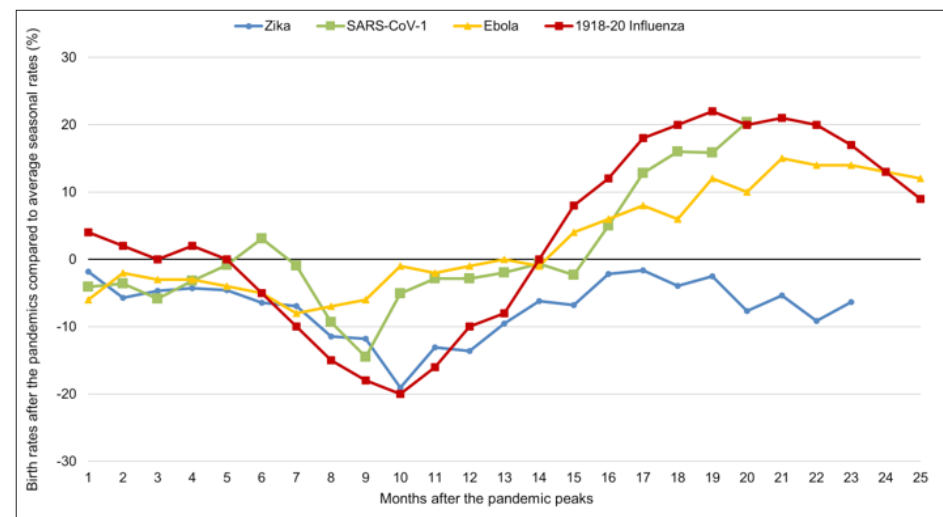

Figure 1: Evolution of birth rates after the previous pandemics, compared to average seasonal rates.

Monthly birth rates were estimated by the number of births per 1000 habitants each month, based on the data available on the UNSD, WHO, CDC, IBRD and IFS Databases. For the 1918-20 Influenza, we grouped data from Switzerland, Denmark, Finland, Sweden, Norway, and United States, and presented the mean. For the Ebola 2015 outbreak, we grouped Guinea, Sierra Leone and Liberia as West Africa. The pandemic peaks were estimated in January 2016 for Zika in Brazil, in May 2003 for SARS in Hong-Kong, in November 2014 for Ebola in West Africa, and in December 1918 for the H1N1 pandemic. Monthly birth rates after the pandemic peaks were compared to average seasonal rates using standardized differences. Standardized differences are presented as percentages in the figure (y). 
Lower birth rates after a global pandemic could be explained by adverse outcomes related to the pathogen (maternal deaths, miscarriages or fetal demise), by the fear of potential complications leading to elective abortions in cases of maternal exposure, by the decision to postpone pregnancies in couples who would otherwise be willing to conceive, as well as herd or population stress which is known to decrease fertility rates. The high global incidence of disease and its widespread media coverage are also factoring that may contribute to an overall decline in births during and in the 9 months after a pandemic. Concerning SARS-CoV2, Chen et al. [6] recently reported four elective abortions in nine (44\%) pregnant women infected during the first trimester [6]. In their series, three other patients $(3 / 9,33 \%)$ lost their pregnancies due to early miscarriages. Other complications, such as fetal growth restriction and subsequent demise could occur later in pregnancy [7]. Cessation of IVF during the crisis is also a factor limiting conceptions (3\% of pregnancies are obtained by IVF) [8]. Several factors that could potentially influence conception and birth during the present pandemic, however, remain difficult to investigate. These include the fertility impact of social distancing and quarantine measures, rates of adverse maternal and fetal outcomes, and access to elective abortions during the pandemic. Nonetheless, by analogy with prior worldwide pandemics, we should expect a drop in global births during and up to 9 months following the conclusion of the pandemic, followed by a subsequent rebound in births as time passes. These changes in birth rate might of course have a direct impact on the supply of maternity services worldwide, including the location and number of healthcare providers.

This decline in birth rates could particularly affect long-term demography in societies where the population growth is already almost negative, such as Western Europe. In Italy for example, a 15-percent drop in births now would result in 2 retirees for each active worker in 20 years. These macro changes in age distribution carry major societal, cultural and economic long-term repercussions. By contrast, they may be less impactful in resource limited settings, where the population pyramid is younger and thus likely to experience less deaths due to COVID-19. In conclusion, when an epidemic turns deadly, the effects on fertility, age distribution, and social structure may persist for decades beyond the immediate period of contagion.

\section{Competing interests}

The authors declare that they have no competing interests

\section{Authors' contributions}

$\mathrm{AC}$ and LP did the literature review, the analyze of datasets, and draw the figure. LP, GF, JH, KNS and DB interpreted the data. All authors participated in the manuscript writing.

\section{References}

1. Dahal S, Mizumoto K, Bolin B, Viboud C, Chowell G (2018) Natality decline and spatial variation in excess death rates during the 1918-1920 influenza pandemic in Arizona, United States. Am J Epidemiol 187(12): 2577-2584.

2. Feshbach KB, Simonsen L, Viboud C, Mølbak K, Miller MA, et al. (2011) Natality decline and miscarriages associated with the 1918 influenza pandemic: the Scandinavian and United States experiences. J Infect Dis 204(8): 1157-1164.

3. Mamelund SE (2004) Can the Spanish influenza pandemic of 1918 explain the baby boom of 1920 in neutral Norway? Population 59(2): 269-301.

4. Helgertz J, Bengtsson T (2019) The long-lasting influenza: the impact of fetal stress during the 1918 influenza pandemic on socioeconomic attainment and health in Sweden, 1968-2012. Demography 56(4): $1389-1425$.

5. Coelho FC, Armstrong M, Saraceni V, Lemos C (2017) Can zika account for the missing babies? Front Public Health 5: 317.

6. Chen L, Li Q, Zheng D, Jiang H, Wei Y, et al. (2020) Clinical characteristics of pregnant women with covid-19 in Wuhan, China. N Engl J Med 382(25): e100.

7. Baud D, Greub G, Favre G, Gengler C, Jaton K, et al. (2020) Secondtrimester miscarriage in a pregnant woman with sars-cov-2 infection. JAMA 323(21): 2198-2200.

8. Vaiarelli A, Bulletti C, Cimadomo D, Borini A, Alviggi C, et al. (2020) COVID-19 and ART: the view of the Italian society of fertility and sterility and reproductive medicine. Reprod Biomed Online 40(6): 755-759. 